\title{
Clinical approach to acute interstitial lung diseases
}

\author{
Luca Bilucaglia \\ Luca Novelli \\ Alice Biffi \\ Alberto Pesci
}

Pneumologic Clinic, "San Gerardo" Hospital, Monza, Italy; University of Milano-Bicocca, Milano, Italy

\author{
Address for correspondence: \\ Alberto Pesci \\ Pneumologic Clinic \\ "San Gerardo" Hospital, Monza \\ University of Milano-Bicocca \\ Milano, Italy \\ E-mail: alberto.pesci@unimib.it
}

\section{Summary}

A subset of patients who present with acute respiratory symptoms will develop acute hypoxic respiratory failure with bilateral lung infiltrates and may fulfill clinical criteria for the acute respiratory distress syndrome.

There is a wide variety of well-known etiologies, including infection/sepsis, shock, trauma, aspiration, oxygen toxicity and many others. A few cases occur without an apparent cause or underlying context such as acute interstitial pneumonia (AIP), cryptogenic organizing pneumonia (COP), acute eosinophilic pneumonia (AEP), while others occur as a rapid deterioration of previously diagnosed or undiagnosed chronic interstitial lung disorders (ILD), such as idiopathic pulmonary fibrosis (IPF), nonspecific interstitial pneumonia (NSIP), hypersensitivity pneumonitis (HP), collagen vascular disease with ILD (CVD-ILD). These patients need a correct setting and management, especially regarding their oxygenation. It's very important to identify cases that have a treatable or potentially reversible cause, and distinguish them from those in whom the etiology is unknown and the response to therapy is likely to be poor. Primarily pulmonary infection, heart failure, pulmonary thromboembolism, drug toxicity and collagen vascular diseases should be excluded.

The most important diagnostic tools are clinical features, radiologic pattern, bronchoalveolar lavage, transbronchial lung biopsy and surgical biopsy. Fast diagnosis and prompt beginning of therapy are of pivotal importance.
KEY WORDS: diffuse parenchymal lung disease, interstitial lung disease, acute interstitial pneumonia, nonspecific interstitial pneumonia, acute respiratory failure, acute exacerbation, idiopathic pulmonary fibrosis, acute eosinophilic pneumonia, organizing pneumonia.

\section{Introduction}

A subset of patients who present with acute respiratory symptoms will develop acute hypoxic respiratory failure with bilateral lung infiltrates. These patients may fulfill clinical criteria for the acute respiratory distress syndrome (ARDS), including acute onset, $\mathrm{PaO}_{2} / \mathrm{Fl}_{02}$ ratio $\leq 300 \mathrm{~mm} \mathrm{Hg}$, bilateral pulmonary infiltrates on chest radiographs and absence of cardiogenic pulmonary edema.

This is a "mixed bag" in terms of etiology and underlying pathology, rather than a well-defined clinicopathological entity (1-3). From an etiologic standpoint, there is a wide variety of well-known settings, including infection/sepsis, shock, trauma, aspiration, oxygen toxicity and many others (4). A few cases occur without an apparent cause or underlying context such as acute interstitial pneumonia (AIP), cryptogenic organizing pneumonia (COP) (5), acute eosinophilic pneumonia (6); while others occur as a rapid deterioration (RD) of previously diagnosed or undiagnosed chronic interstitial lung disorders (ILD), such as idiopathic pulmonary fibrosis (IPF) (7-9), nonspecific interstitial pneumonia (NSIP) (10), hypersensitivity pneumonitis (HP) (11), colla-
Acute exacerbation of IPF (AEx - IPF) is the prototype and best studied acute exacerbation (AEx) of ILD (AEx-ILD). gen vascular disease with ILD (CVD-ILD) $(10,12)$. The prototype and best studied of these miscellaneous conditions is the acute exacerbation of IPF (AEx - IPF), and along our manuscript we will mainly refer to AExIPF.

The challenge for the clinician managing patients with acute respiratory failure is to identify cases that have a treatable or potentially reversible cause, and distinguish them from those in whom the etiology is unknown and the response to therapy is likely to be poor.

\section{Patient setting}

Correct oxygenation has a pivotal role in the management of these patients. The likelihood of a successful outcome is the key consideration that should guide a decision to admit patients with acute ILD to an inten- 
sive care unit (ICU) for invasive mechanical ventilation (MV). It is more appropriate to perform a trial of noninvasive ventilation (NIV) in an intermediate respiratory unit before consider patient ICU access. These patients should carefully monitored and not hospitalized in general ward. MV may seem an attractive option when the disease is potentially reversible. Guidelines concerning the referral to the ICU and initiation of MV are lacking, and evidences suggest that invasive MV for patients with acute ILD is appropriate when respiratory failure follows a surgical procedure but is questionable in other circumstances because the outcome of these patients admitted in ICU is very poor and MV is mostly futile. In hospital mortality rate of patient with AEx-IPF mechanically ventilated in ICU is always very elevated, among $80-100 \%(13-15)$. This has led many intensivists to deny initiation of invasive MV in this setting.

NIV approach seems to have lower mortality due, maybe, to less deteriorated patients and less baro/volutrauma and ventilator induced lung injury (VILI) (15, 16).

$\mathrm{MV}$ is increasingly regarded as an absolute contraindication to lung transplantation, because in these cases the outcome is poor due to an increased risk of pneumonia, caused by airways microbial colonization associated, of severe muscular deconditioning, depending on a protracted immobility, and of complications such as sepsis and nutritional problems. New strategies of bridging critical candidates to lung transplantation, like extracorporeal membrane oxygenation (ECMO) support, are promising strategies (17).

\section{Clinical approach}

There is not a standardized approach in diagnostic workup of these patients. Wuyts et al. (18) proposed a diagnostic algorithm, but it needs validation in prospective studies. We propose the diagnostic algorithm used at our institution in the management of acute presentation of interstitial lung disease (Figure 1).

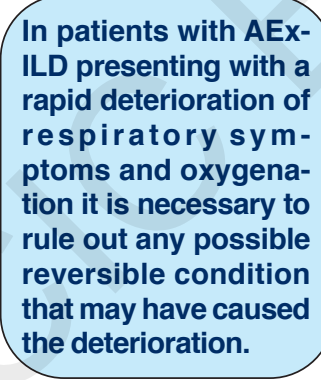

The exclusion of underlying occult etiologies becomes an issue. Some causes of ARDS, such as sepsis, prior chemotherapy, surgery, aspiration, or massive trauma are clinically obvious at the time of diagnosis.

In patients presenting with a rapid deterioration of respiratory symptoms and oxygenation it is necessary to rule out any possible reversible condition that may have caused the deterioration: pulmonary infection, heart failure and pulmonary thromboembolism, as principals in decreasing order of frequency (8). However, other less frequent etiologies such as drug toxicity or connective tissue diseases should be rule out. It is important to perform all the diagnostic examinations as soon as possible because a fast diagnostic approach resulting in a precocious starting of therapy was associated with a lower in hospital mortality (19). The delay of some diagnostic examinations could lead to an impossibility to perform them (e.g. bronchoscopy) because of rapid clinical deterioration.

\section{Diagnostic tools}

The most important diagnostic tools are clinical features, radiologic pattern, bronchoalveolar lavage, transbronchial lung biopsy and surgical biopsy.

\section{Clinical features}

Patients complain rapid onset of worsening dyspnea with different degrees of cough and sputum production. Sometimes there is fever or others flu-like
BAL must be performed soon after admission and its analysis is fundamental to exclude infection. Distinctive findings in BAL fluid cytology may help diagnosis of non-infectious conditions. symptoms. All these symptoms are not specific and cannot guide unambiguously differential diagnosis. There are few symptoms or signs that according to previous history can help differential diagnosis [e.g. orthopnea with peripheral edema, hepatogiugular reflux and an history of cardiac impairment can suggest a diagnosis of congestive heart failure (CHF)]. A dyspnea began in a precise moment, associated to pleural/thoracic pain, tachycardia, and an history of previous DVT can be associated to pulmonary embolism (PE). Specific systemic involvement such as rheumatoid nodules, cutaneous manifestations (e.g. finger cutaneous thickening, puffy hands, mechanic hands) or osteo-articular deformities (e.g. ulnar deviation, boutonniere deformity, swan neck deformity) can lead to a possible diagnosis of CVD-ILDs. Moreover, the presence of digital clubbing means that patient already suffered from chronic respiratory disease.

\section{Radiology}

A chest $\mathrm{x}$-ray is performed at first instance, showing bilateral pulmonary infiltrates. This aspect is not specific for any etiology; however, it can be useful to rule out pneumothorax. After chest x-ray, a chest HRCT is performed, combined with angio-CT if the suspicion of PE hasn't been ruled out.

HRCT in AEx-IPF and other AE-ILDs generally demonstrates bilateral ground glass opacities (GGO) with or without areas of consolidation, superimposed on the fibrotic signs $(8,20)$ (Figure 2a, b). The distribution of GGO can be peripheral, multifocal or diffuse $(20,21)$. Underlying fibrotic abnormalities could be representative of a chronic illness, but appreciation of these signs (honeycombing, traction bronchiectasis, reticular pattern) in an acute context of diffuse GGO +/- consolidation is, sometimes, very difficult. Radiologically ARDS can be indistinguishable from cardiogenic pulmonary edema (22), and infective etiology cannot be distin- 


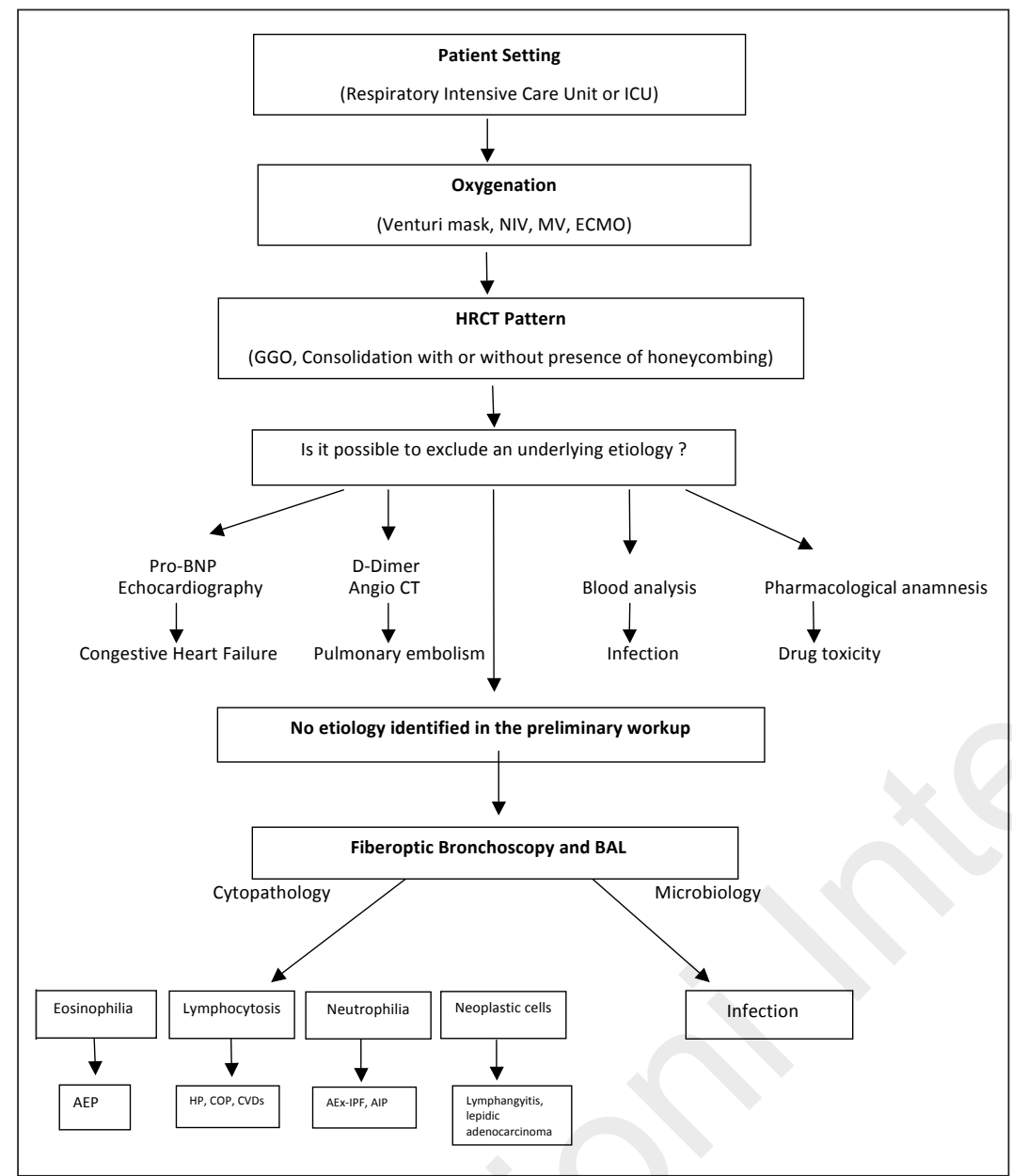

Figure 1 - Clinical approach to acute ILD with respiratory failure.

AEP: Acute eosinophilic pneumonia, HP: Hypersensitivity pneumonitis, CVDs: Collagen vascular diseases, AEx-IPF: Acute exacerbation of idiopathic pulmonary fibrosis, AIP: Acute interstitial pneumonia ICU: Intensive care unit, NIV: Non invasive ventilation, MV: Mechanical ventilation, ECMO: ExtraCorporeal Membrane Oxygenation, GGO: Ground glass opacity

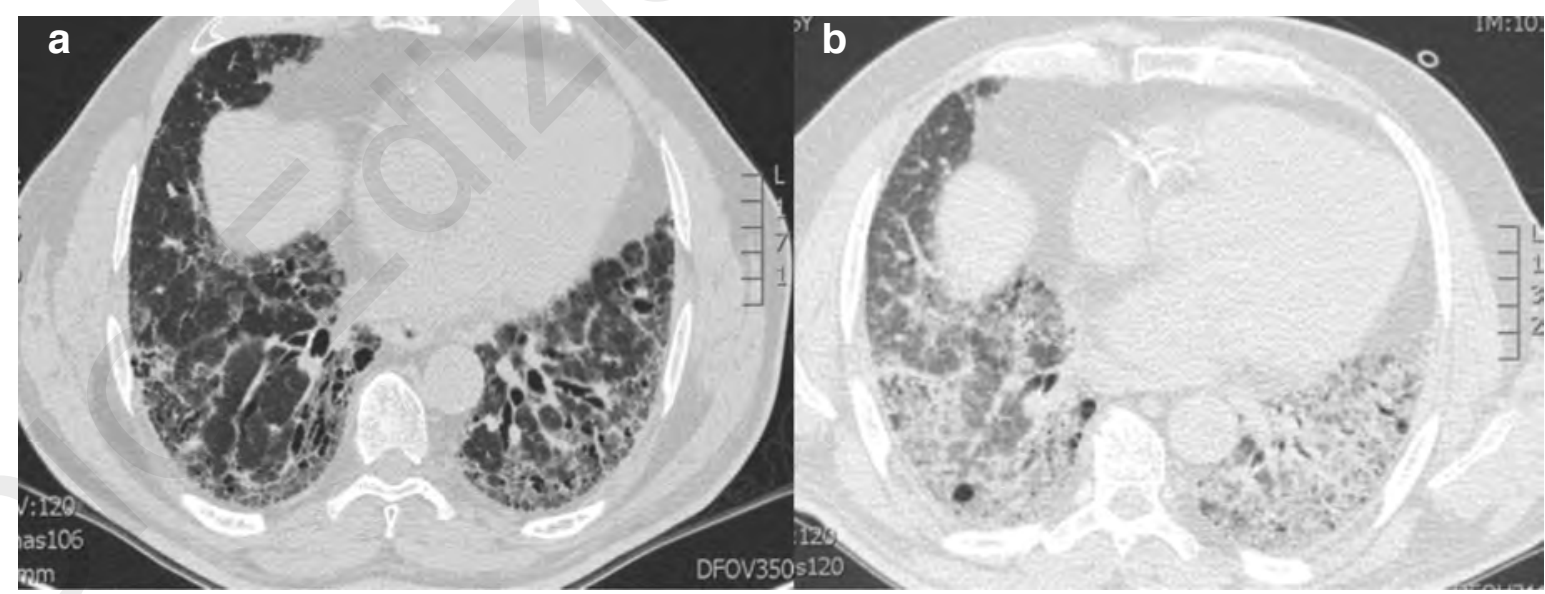

Figure $2 \mathrm{a}, \mathrm{b}$ - Radiological pattern of AEx-IPF.

a. Reticular abnormalities mainly subpleural and basal with traction bronchiectasis and minimal honeycombing.

b. Newly appeared parenchymal bilateral abnormalities: groundglass attenuations and diffuse consolidations.

guished from AEx-ILD. Chest HRCT is also important to guide the invasive diagnostic approach.

\section{Bronchoalveolar lavage}

BAL must be performed soon after admission and its analysis is fundamental to exclude infection. Moreover distinctive findings in BAL fluid cytology, such as lymphocytosis, the presence of activated lymphocytes, plasma cells and eosinophils or the preponderance of foamy macrophages can point to drug-induced pul- 


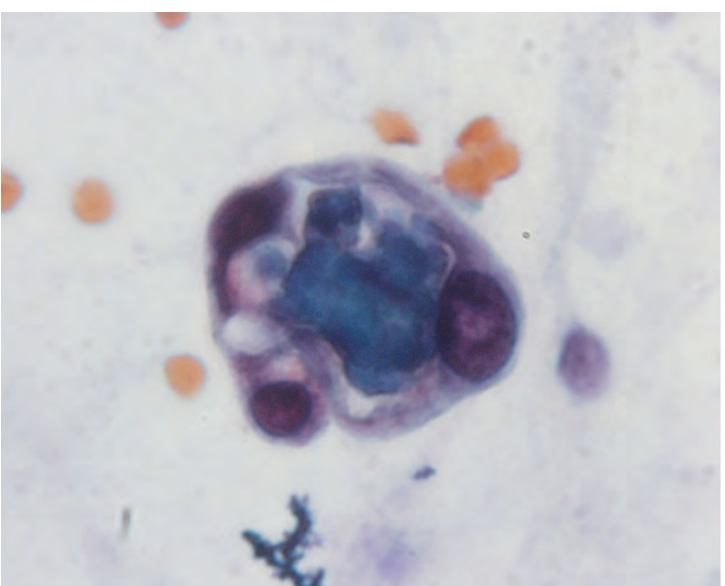

Figure 3 - Cell pellet from BAL of patient with ARDS: we can observe the presence of reactive type II pneumocytes with amorphous material, their atypia mimics carcinoma (MGG 1000X).

monary disease $(23,24)$. The presence of reactive type II pneumocytes has been described in BAL (Figure 3) of patients with DAD (25), their atypia may be severe enough to mimic carcinoma (26). BAL fluid cytological findings in DAD are characterized by a marked predominance of neutrophils in the early phase and a recruitment of macrophages, lymphocytes and eosinophils in the late phase (27). A number of more than $20 \%$ of hemosiderin laden macrophages has been demonstrated to be indicative for diffuse alveolar haemorrhage (28). Analysis of BAL fluid in acute eosinophilic pneumonia shows a very high percentage (more than 25\%) of eosinophils (29), in this case, BAL eosinophilia obviates the need for lung biopsy for the diagnosis $(29,30)$.

However, the usefulness of BAL fluid cytology in the diagnosis of these non-infectious pulmonary conditions in critical patients has received little attention in the literature (31), probably because of the cytological findings under consideration are not pathognomonic. We can observe alveolar hemorrhage in BAL fluid samples from bacterial pneumonia (28), foamy macrophages and plasma cells similar to cases of drug-induced toxicity from patients with $P$. jirovecii pneumonia, and elevated numbers of lymphocytes in BAL fluid from patients with tuberculosis (32). These observations emphasize the importance of perform microbiological investigation, even if BAL fluid cytology at first glance points to a non-infectious condition.

\section{Transbronchial Lung Biopsy (TLB) and Surgical Biopsy (SLB)}

TLB and SLB are potentially dangerous methodology (oxygen deterioration, bleeding, pneumothorax) in patients with ARDS, but are justifiable in certain cases (e.g., no previous diagnosis of ILD, young patients). The decision should be based on clinical scenario. Usually, histological evaluation permits the diagnosis of diffuse alveolar damage (DAD) and/or BOOP, but does not offer additional information on how to cure the patient.

\section{Acute ILD of known causes}

Drugs. Diffuse alveolar damage (DAD) is a commonly reported histological manifestation of drug toxicity (33). Many drugs can cause DAD (e.g., chemotherapeutic agents such as bleomycin and busulfan and nonchemotherapeutic agents such as amiodarone and nitrofurantoin). Drug-related lung disease is always a complicated diagnosis that is difficult if not impossible to prove. In most cases, a presumptive diagnosis of drug toxicity is based on the onset of disease after beginning of drug therapy and improvement of symptoms with cessation of therapy. It is important to stress that no specific
DAD usually occurs in patients with established disease, and is discovered either at with other systemic course of the illness. presentation along features, or later in the pathological findings are unique to drug-related lung disease, or pathognomonic of any specific drug (34).

\section{Collagen Vascular Diseases (CVDs)}

The CVDs that are mainly associated with ARDS/DAD pattern are dermatomyositis/polymyositis (including the antisynthetase syndrome), systemic sclerosis, systemic lupus erythematosus, Sjögren syndrome, rheumatoid arthritis, and mixed connective tissue disease $(35,36)$. DAD usually occurs in patients with established disease, and is discovered either at presentation along with other systemic features, or later in the course of the illness. However, it can occasionally be the presenting manifestation of the disease $(37,38)$. The clinical examination and the required serological tests are the mainstay of the diagnosis.

\section{Infections}

It is a difficult challenge to exclude respiratory infection because of the similarity between clinical features of infection and AEx-ILDs. We can usually perform microbiological tests on serum and urine, however, the more sensibility and specificity is obtained on BAL specimens $(7,19,39)$.

\section{Congestive Heart Failure}

The diagnosis of CHF can be made reliably with the use of various noninvasive tools such as serum B-type natriuretic peptide (BNP) levels and echocardiography. Echocardiography identifies overt cardiac decompensation or valvular heart disease, permitting at the same time the exclusion of pulmonary hypertension.

\section{Pulmonary embolism}

Pulmonary embolism can be ruled out with the combined use of D-dimer serum levels and/or angio CT scan (40).

\section{Acute ILD of unknown causes}

\section{Acute Interstitial Pneumonia (AIP)}

AIP is a rare and fulminant form of idiopathic interstitial pneumonia, with acute onset and rapidly progressive 
course (41). AIP affects healthy individuals, with a mean age of 50 years-old. Prodromal illness typically lasts 7-14 days with fever, cough, and progressive, severe shortness of breath (42). Most patients develop severe hypoxemia with ARDS (43). AIP has histopathologic appearance of DAD with temporally uniform lesions $(42,43)$. Following the acute phase, a stage of organization is characterized by fibroblast proliferation and connective tissue synthesis. The chest imaging findings are similar to ARDS, revealing bilateral, patchy, symmetric areas of ground glass attenuation, often accompanied by airspace consolidation and septal thickening (42-44). The in-hospital mortality from AIP is greater than $50 \%$ and the majority of those who survive dies within six months of presentation. Development of chronic interstitial lung disease have been also reported (45).

\section{Acute exacerbation of IPF (AEX-IPF)}

AEx IPF is defined as a rapid deterioration of IPF in which all the following criteria are met (7):

- Previous or concurrent diagnosis of IPF

- Unexplained worsening or development of dyspnea within 30 days

- HRCT with new bilateral ground glass abnormality and/or consolidation superimposed on a background reticular or honeycomb pattern consistent with UIP pattern

- No evidence of pulmonary infection by endotracheal aspirate or BAL

- Exclusion of alternative cause, including left heart failure, pulmonary embolism, identifiable cause of acute lung injury.

The 1-yr incidence varies between 5-14.2\% (7-9, 4648), 3-yr incidence between $20.7-24 \%(8,48)$. Gender ratio seems to be $4: 1,2: 1$ for men $(8,19,49)$ and patients are in their 60-70's $(8,19)$. Intrahospital mortality is more than $50 \%,(8,49), 3$ months mortality between $60-64 \%(8,49)$, and in one study $1-y r$ mortality reaches $100 \%(50)$. Median survival is between 2.29.3 months $(8,19,20,49)$.

It remains unclear whether $A E$ represents a primary acceleration of the underlying fibroproliferative process

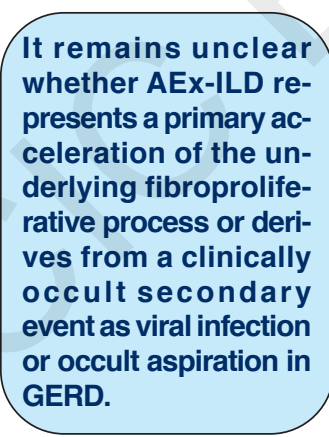
or derives from a clinically occult secondary event as occult viral infection (51) or occult aspiration in GERD (52). Precipitating factors have been recognized in surgical lung biopsy or other lung surgery (53), BAL (54), air pollution exposure (55). HRCT generally demonstrates bilateral ground glass abnormalities with or without areas of consolidation, superimposed on the IPF abnormalities (21, 39), with peripheral, multifocal or diffuse distribution. Superimposed on UIP pattern, the most common histopathological finding is DAD, in a minority of cases also OP and extensive fibroblastic foci $(21,39)$. The prognosis seems to be better in the last two cases
(20). An increase in BALF neutrophils has also been suggested, however, lymphocytosis can be seen (39).

\section{Cryptogenic Organizing Pneumonia (COP)}

Patients with COP typically present with a subacute illness of relatively short duration with cough and dyspnea $(56,57)$. COP is defined pathologically by the presence in the distal air spaces of buds of granulation tissue progressing from fibrin exudates to loose collagen containing fibroblasts. The lesions occur predominantly within the alveolar spaces but are often associated with buds of granulation tissue occupying the bronchiolar lumen (bronchiolitis obliterans) called Masson's bodies (58). HRCT characteristically demonstrates patchy and often migratory consolidation in a subpleural, peribronchial, or band-like pattern, commonly associated with ground-glass opacity (57). The majority of patients recover completely with oral corticosteroids, but relapse is common $(56,57)$. The BAL cell count may show a mixed alveolitis with increased lymphocytes (>20\%) with CD4/CD8 ratio decreased, neutrophils (<10\%) and eosinophils (about 5\%) (56).

\section{Acute eosinophilic pneumonia (AEP)}

AEP rapidly progress from mild-to-severe hypoxaemic respiratory failure requiring intensive care. Pleuritic chest pain and myalgia may be present in over half of all patients. The average age at presentation is 30 years-old and there is no gender preference. A smoking history is present in $40 \%$ of patients $(6,59)$. Unlike other eosinophilic pneumonias, patients with idiopathic AEP usually have normal or only slightly elevated peripheral blood eosinophil counts (6). BAL is important in excluding bacterial, fungal, and other infections, and characteristically shows more than $25 \%$ of eosinophils (60). Histological findings include infiltration of eosinophils in the interstitium and in the alveolar spaces with features of diffuse alveolar damage (61). The HRCT findings include diffuse areas of groundglass attenuation, nodules, smooth interlobular septal thickening; small-to-moderate pleural effusions are frequent (62).

\section{Treatment}

High dose glucocorticoid treatment is efficacious in COP, AEP, drug toxicity and CVDs. An evidence-based approach to the therapy of AIP and AEx-IPF is very difficult because of the rarity of the diagnosis and because all reports of these conditions to date are small and descriptive case series. The benefit of glucocorticoids remains unclear, although these are widely used $(7,9,42)$. Alternative immunosuppressive therapies (e.g., cyclophosphamide, cyclosporine and azathioprine) have been reported in case series with
An evidence-based approach to the therapy of AIP and AExIPF is very difficult of the diagnosis and because all reports of these conditions to date are small and descriptive case series. because of the rarity 
doubtful results (63). In very selected cases lung transplantation remains the unique option.

\section{References}

1. Schwarz MI, Albert RK. "Imitators" of the ARDS: implications for diagnosis and treatment. Chest. 2004;125:1530-1535.

2. Ranieri VM, Rubenfeld GD, Thompson BT, Ferguson ND, Caldwell E, Fan E, Camporota, et al. Acute respiratory distress syndrome: the Berlin Definition. JAMA. 2012;307(23):2526-33.

3. De Hemptinne $Q$, Remmelink $M$, Brimioulle $S$, Salmon I, Vincent JL. ARDS: a clinicopathological confrontation. Chest. 2009;135:944-949.

4. Katzenstein AL, Bloor CM, Leibow AA. Diffuse alveolar damage - the role of oxygen, shock, and related factors: a review. Am J Pathol. 1976;85:209228.

5. Travis WD, Costabel U, Hansell DM, King TE Jr, Lynch DA, Nicholson AG, et al. An official American Thoracic Society/European Respiratory Society statement: update of the international multidisciplinary classification of the idiopathic interstitial pneumonias. Am J Respir Crit Care Med. 2013;14:733748.

6. Janz DR, O'Neall HR, JR, Ely EW. Acute eosinophilic pneumonia: A case report and review of the literature. Crit Care Med. 2009;37:1470-1474.

7. Collard HR, Moore BB, Flaherty KR, et al. Acute exacerbation of idiopathic pulmonary fibrosis. Am J Respir Crit Care Med. 2007;176:636-643.

8. Song JW, Hong SB, Lim CM, et al. Acute exacerbation of idiopathic pulmonary fibrosis: incidence, risk factors and outcome. Eur Respir J. 2011;37:356363.

9. Kim DS. Acute exacerbation of idiopathic pulmonary fibrosis. Clin Chest Med. 2012;33:59-68.

10. Park IN, Kim DS, Shim TS, Lim CM, Lee SD, Koh Y, $\mathrm{Kim}$, et al. Acute exacerbation of interstitial pneumonia other than idiopathic pulmonary fibrosis. Chest. 2007;132:214-220.

11. Miyazaki $Y 1$, Tateishi $T$, Akashi $T$, Ohtani $Y$, Inase $N$, Yoshizawa Y. Clinical predictors and histologic appearance of acute exacerbations in chronic hypersensitivity pneumonitis. Chest. 2008;134:1265-70.

12. Tachikawa R, Tomii K, Ueda H, Nagata K, Nanjo S, Sakurai $A$, et al. Clinical features and outcome of acute exacerbation of interstitial pneumonia: collagen vascular diseases-related versus idiopathic. Respiration. 2012;83:20-7.

13. Al-Hameed FM, Sharma S. Outcome of patients admitted to the intensive care unit for acute exacerbation of idiopathic pulmonary fibrosis. Can Respir J. 2004;14:117-122

14. Mollica C, Paone G, Conti V, Ceccarelli D, Schmid G, Mattia $P$, et al. Mechanical ventilation in patients with end-stage idiopathic pulmonary fibrosis. Respiration. 2010;79:209-215.

15. Gaudry S, Vincent F, Rabbat A, Nunes H, Crestani B, Naccache JM, et al. Invasive mechanical ventilation in patients with fibrosing interstitial pneumonia. $J$ Thorac Cardiovasc Surg. 2014;147:47-53.

16. Vianello A, Arcaro G, Battistella L, Pipitone E, Vio S, Concas $A$, et al. Noninvasive ventilation in the event of acute respiratory failure in patients with idiopathic pulmonary fibrosis. J Crit Care. 2014 Mar 30. pii: S0883-9441(14)00119-1. [Epub ahead of print].

17. Fuehner T, Kuehn C, Hadem J, Wiesner O, Gottlieb $\mathrm{J}$, Tudorache I, et al. Extracorporeal membrane oxygenation in awake patients as bridge to lung transplantation. Am J Respir Crit Care Med. 2012;185: 763-8.

18. Wuyts WA, Thomeer M, Dupont LJ, Verleden GM. An algorithm to tackle acute exacerbations in idiopathic pulmonary fibrosis. Am J Respir Crit Care Med. 2008;177:1397.

19. Simon-Blancal V, Freynet O, Nunes H, Bouvry D, Naggara N, Brillet PY, Denis D, et al. Acute exacerbation of idiopathic pulmonary fibrosis: outcome and prognostic factors. Respiration. 2012;14:28-35.

20. Silva Cl, Müller NL, Fujimoto K, et al. Acute exacerbation of chronic interstitial pneumonia: high-resolution computed tomography and pathologic findings. J Thorac Imaging. 2007;22:221-9.

21. Akira M, Hamada H, Sakatani M, Kobayashi C, Nishioka M, Yamamoto S. CT findings during phase of accelerated deterioration in patients with idiopathic pulmonary fibrosis. AJR Am J Roentgenol. 1997;168: 79-83.

22. Ware LB, Matthay MA. The acute respiratory distress syndrome. N Engl J Med. 2000;342:1334-1349.

23. Drent $M$, van Velzen-Blad $H$, Diamant $M$, Wagenaar $\mathrm{S}$, Donkerwolcke-Bogaert M, van den Bosch JMM. Differential diagnostic value of plasma cells in bronchoalveolar lavage fluid. Chest. 1993;103:17201724.

24. Rosenow EC III, Martin WJ II. Drug-induced interstitial lung disease. In: Schwarz MI, King TE (eds). Interstitial Lung Disease. 2nd edn, 1993:255-270.

25. Grotte D, Stanley MW, Swanson PE, Henry-Stanley $J$, Scott D. Reactive Type II pneumocytes in bronchoalveolar lavage fluid from adult respiratory distress syndrome can be mistaken for cells of adenocarcinoma. Diagn Cytopathol. 1990;6:317-322.

26. Biyoudi-Vouenze R, Tazi A, Hance AJ, Chastre J, Basset F, Soler P. Abnormal epithelial cells recovered by bronchoalveolar lavage: Are they malignant? Am Rev Respir Dis. 1990;142:686-690.

27. Nakos G, Kitsiouli EL, Tsangaris L, Lekka MD. Bronchoalveolar lavage fluid characteristics of early intermediate and late phases of ARDS. Intensive Care Med. 1988;24:296-303.

28. Lassence de A, Fleury-Feith J, Escudier E, Beaune J, Bernaudin JF, Cordonnier C. Alveolar hemorrhage. Diagnostic criteria and results in 194 immunocompromised hosts. Am J Respir Crit Care Med. 1995;151: 157-163.

29. Pope-Harman AL, Davis WB, Allen ED, Christoforidis AJ, Allen JN. Acute eosinophilic pneumonia. A summary of 15 cases and review of the literature. Medicine. 1996;75:334-342.

30. Philit F, Etienne-Mastroianni B, Parrot A, Guerin C, 
Robert D, Cordier JF. Idiopathic acute eosinophilic pneumonia: a study of 22 patients. Am J Respir Crit Care Med. 2002;166:1157-1158.

31. Jacobs JA, De Brauwer EIGB, Ramsay G, et al. Detection of non-infectious conditions mimicking pneumonia in the intensive care setting: usefulness of bronchoalveolar fluid cytology. Respir Med. 1999; 93:571-578.

32. Baughman RP, Dohn MN, London RG, Frame PT. Bronchoscopy with bronchoalveolar lavage in tuberculosis and fungal infections. Chest. 1991;99:92-97.

33. Myers JL, Limper AH, Swensen SJ. Drug-induced lung disease: a pragmatic classification incorporating HRCT appearances. Semin Respir Crit Care Med. 2003;24:445-454.

34. Camus P, Fanton A, Bonniaud P, Camus C, Foucher $P$. Interstitial lung disease induced by drugs and radiation. Respiration. 2004;71:301-326.

35. Fischer A, West SG, Swigris JJ, Brown KK, du Bois RM. Connective tissue disease-associated interstitial lung disease: a call for clarification. Chest. 2010; 138:251-256.

36. Parambil JG, Myers JL, Ryu JH. Diffuse alveolar damage: uncommon manifestation of pulmonary involvement in patients with connective tissue diseases. Chest. 2006;130:553-558.

37. Parambil JG, Myers JL, Lindell RM, Matteson EL, Ryu JH. Interstitial lung disease in primary Sjögren syndrome. Chest. 2006;130:1489-1495.

38. Douglas WW, Tazelaar HD, Hartman TE, et al. Polymyositis-dermatomyositis-associated interstitial lung disease. Am J Respir Crit Care Med. 2001;164: 1182-1185.

39. Kim DS, Park JH, Park BK, Lee JS, Nicholson AG, Colby T. Acute exacerbation of idiopathic pulmonary fibrosis: frequency and clinical features. Eur Respir J. 2006;27:143-150.

40. Roy PM, Colombet I, Meyer G, et al. Systematic review and meta-analysis of strategies for the diagnosis of suspected pulmonary embolism. BMJ. $2005 \mathrm{Jul}$ 30;331(7511):259.

41. Olson J, Colby TV, Elliott CG. Hamman-Rich syndrome revisited. Mayo Clin Proc. 1990;65:1538-48.

42. Vourlekis JS. Acute interstitial pneumonia. Clin Chest Med. 2004;25:739-47.

43. Fulmer JD, Katzenstein ALA. The interstitial lung diseases. In: Pulmonary and Critical Care Medicine, Bone RC (Ed), Mosby Year Book, St. Louis 1993. p.M1.

44. Bouros D, Nicholson AC, Polychronopoulos V, du Bois RM. Acute interstitial pneumonia. Eur Respir J. 2000;15:412-418.

45. Vourlekis JS, Brown KK, Cool CD, et al. Acute interstitial pneumonitis. Case series and review of the literature. Medicine (Baltimore). 2000;79:369-78.

46. Taniguchi $\mathrm{H}$, Ebina $\mathrm{M}, \mathrm{Kondoh} \mathrm{Y}$, et al. Pirfenidone in idiopathic pulmonary fibrosis. Eur Respir J. 2010;35:821-829.

47. Azuma A, Nukiwa T, Tsuboi E, et al. Double-blind, placebo-controlled trial of pirfenidone in patients with idiopathic pulmonary fibrosis. Am J Respir Crit Care Med. 2005;171:1040-1047.
48. Kondoh $\mathrm{Y}$, Taniguchi $\mathrm{H}$, Katsuta T, Kataoka K, Kimura T, Nishiyama O, et al. Risk factors of acute exacerbation of idiopathic pulmonary fibrosis. Sarcoidosis Vasc Diffuse Lung Dis. 2010;14:103-110.

49. Kishaba T, Tamaki H, Shimaoka Y, Fukuyama H, Yamashiro S. Staging of acute exacerbation in patients with idiopathic pulmonary fibrosis. Lung. 2014; 192:141-9.

50. Huie TJ, Olson AL, Cosgrove GP, Janssen WJ, Lara AR, Lynch DA, et al. A detailed evaluation of acute respiratory decline in patients with fibrotic lung disease: aetiology and outcomes. Respirology. 2010;14: 909-917.

51. Wootton SC, Kim DS, Kondoh Y, Chen E, Lee JS, Song JW, Huh, et al. Viral infection in acute exacerbation of idiopathic pulmonary fibrosis. Am J Respir Crit Care Med. 2011;14:1698-1702.

52. Lee JS, Song JW, Wolters PJ, Elicker BM, King TE Jr, Kim DS, Collard HR. Bronchoalveolar lavage pepsin in acute exacerbation of idiopathic pulmonary fibrosis. Eur Respir J. 2012;1 4:352-358.

53. Kondoh $\mathrm{Y}$, Taniguchi $\mathrm{H}$, Kitaichi M, Yokoi T, Johkoh T, Oishi T, Kimura T, et al. Acute exacerbation of interstitial pneumonia following surgical lung biopsy. Respir Med. 2006;100:1753-1759.

54. Sakamoto K, Taniguchi $\mathrm{H}$, Kondoh $\mathrm{Y}$, Wakai K, Kimura T, Kataoka K, et al. Acute exacerbation of IPF following diagnostic bronchoalveolar lavage procedures. Respir Med. 2012;14:436-442.

55. Johannson KA, Vittinghoff E, Lee K, Balmes JR, Ji W, Kaplan GG, et al. Acute exacerbation of idiopathic pulmonary fibrosis associated with air pollution exposure. Eur Respir J. 2014;43:1124-31.

56. King TE Jr, Mortenson RL. Cryptogenic organizing pneumonitis: the North American experience. Chest. 1992;102(1, Suppl):8S-13S.

57. Oymak FS, Demirbaş HM, Mavili E, Akgun H, Gulmez I, Demir R, et al. Bronchiolitis obliterans organizing pneumonia: clinical and roentgenological features in 26 cases. Respiration. 2005;72: 54-262.

58. Sulavik SB. The concept of "organizing pneumonia". Chest. 1989;96:967-969.

59. Allen JN, Pacht ER, Gadek JE, Davis WB. Acute eosinophilic pneumonia as a reversible cause of noninfectious respiratory failure. $\mathrm{N}$ Engl J Med. 1989;321:569-74.

60. Shintani H, Fujimura M, Ishiura Y, Noto M. A case of cigarette smoking-induced acute eosinophilic pneumonia showing tolerance. Chest. 2000;117:277-79.

61. Mann B. Eosinophilic Lung Disease. Clinical Medicine: Circulatory, Respiratory and Pulmonary Medicine. 2008;2:99-108.

62. Johkoh T, Muller NL, Akira M, Ichikado K, Suga M, Ando $\mathrm{M}, \mathrm{Y}$, et al. Eosinophilic lung diseases: diagnostic accuracy of thin-section CT in 111 patients. Radiology. 2000;216:773-80.

63. Morawiec E, Tillie-Leblond I, Pansini V, Salleron J, Remy-Jardin M, Wallaert B. Exacerbations of idiopathic pulmonary fibrosis treated with corticosteroids and cyclophosphamide pulses. Eur Respir J. 2011 Dec;38(6):1487-9. 